Muona, O. (1990): Population genetics in forest tree improvement. In: Brown A. H. D., CleGG M. T., KAHLER A. L. and WeIR B. S. (eds.), Plant Population Genetics, Breeding, and Genetic Resources, 282-298. Sinauer, Sunderland, Massachusetts.

NAGANO, S. and J. NASU (1991): Studies on the genetic diversity of Fagus crenata Blume II. Bulletin of the Iwate University Forest 22: 21-28 (in Japanese with English summary).

NAGANO, S., J. NASU and N. Mishima (1990): A study on the genetic diversity of beech forests I. Transactions of the Tohoku Branch meeting of the Japanese Forestry Society 42: 235-237 (in Japanese; the title was translated by the authors).

NEI, M. (1987): Molecular Evolutionary Genetics (Translated by Gojobori. T. \& Saitou. N.). Columbia University Press, New York. 512 pp.

OHKaWA, T., Y. NAGAI, J. MAsuda, K. Kitamura and S. KaWANO (1998): Population biology of Fagus crenata Blume I. Demographic genetic differentiations of lowland and montane populations in Toyama, Central Honshu, Japan. Plant Species Biology 13: 93-116.

OHSAWA, M. (1991): Structural comparison of tropical montane rain forests along latitudinal and altitudinal gradients in South and East Asia. Plant Ecology 97: $1-10$.

ORsteIN, L. (1964): Disk electrophoresis I: background and theory. Annals of New York Academy for Science 121: 321-349.

SOKAL, R. R. and D. L. ODEN (1978a): Spatial autocorrelation in biology. 1. Methodology. Biological Journal of the Linnean Society 10: 199-228.

SoKAL, R. R. and D. L. ODEN (1978b): Spatial autocorrelation in biology. 2. Some biological implications and four applications of evolutionary and ecological interest. Biological Journal of the Linnean Society 10: 229-249.

Silverstown, J. W. and J. L. Doust (1993): Introduction to plant population biology, $3^{\text {rd }}$ ed. 210 pp. Blackwell, London.
SHIRAISHI, S. (1988): Inheritance of isozyme variation in Japanese black pine, Pinus thunberigii Parl. Silvae Genetica 37: 93-100.

Slate, J., T. Marshall and J. Pemberton (2000): A retrospective assessment of the accuracy of the paternity inference program CERVUS. Molecular Ecology 9: 801-808.

TAKAHASHI, M. (2003): PSAwinD version 1.1.1: a program for calculating spatial indices. Journal of Heredity 94: 267-270.

TAKaHASHI, M., Y. Tsumura, T. NAKAmURA, K. UChIDA and K. OHBA (1994): Allozyme variation of Fagus crenata in northeastern Japan. Canadian Journal of Forest Research 24: 1071-1074.

TAKAHAShI, M., M. Mukouda and K. Koono (2000): Differences in genetic structure between two Japanese beech (Fagus crenata Blume) stands. Heredity 84: 103-115.

TAKAHAShi, M., N. Tomaru, M. Ubukata and K. KoONo (2002): Genetic structure in the northernmost marginal population of Japanese beech (Fagus crenata Blume): influence of the founding event on genetic structure. Silvae Genetica 51: 219-225.

TAKIYA, M. and N. HAGIWARA (1997): Vegetational history of Mt. Yokotsudake, southwestern Hokkaido, since the last Glacial. The Quaternary Research 36: 217-234. (in Japanese with English summary).

TATEWAKI, M. (1958): Forest ecology of the islands of the North Pacific Ocean. Journal of the Faculty of Agriculture of Hokkaido University 50: 271-486.

Tomaru, N., T. Mitsutsuji, M. TAKahashi, Y. Tsumura, K. UCHIDA and K. OнвA (1997): Genetic diversity in Fagus crenata (Japanese beech): influence of the distributional shift during the last-Quaternary. Heredity 78: 241-251.

TsukAHARA, M. and H. Miguchi (2004): Effectivity of thinning secondary beech forest. Growth processes for 10 years. Bulletin of Niigata Prefectural Forest Research Institute 45: 17-22. (in Japanese; the title was translated by the authors).

\title{
Age Trends in Genetic Parameters for Growth and Resin-Yielding Capacity in Masson Pine
}

\author{
By L. H. ZenG ${ }^{1)^{*},}$, Q. ZhANG ${ }^{1)}$, B. X. $\mathrm{HE}^{1)}$, H. M. LIAN ${ }^{1)}$, Y. L. $\mathrm{CAI}^{1)}$, Y. S. WANG ${ }^{2)}$ and M. LuO ${ }^{2)}$
}

(Received $18^{\text {th }}$ January 2012)

\begin{abstract}
Masson pine (Pinus massoniana Lamb) has long been employed as a main source of pine resin in China. To get a better understanding of genetic regulation of resin-

1) Guangdong Academy of Forestry, Guangzhou, China, 510520 .

2) Xinyi Forestry Research Institute, Maoming, China, 525300.

*) Corresponding author: LinghaI Zeng. Phone and Fax: +86-2087035645, E-Mail: zlinghai@263.net
\end{abstract}

yielding capacity (RYC), a total of 50 open-pollinated families of masson pine were planted at three testing sites for progeny testing. Investigation was conducted at ages $7,9,11,13,15,20,24$ and 26 years to study inheritance, age-age genetic correlation, and early selection efficiency for RYC, height (HT), diameter at breast height (DBH) and volume of individual tree (VOL). Growth characteristics increased gradually with age. RYC had a rapid increase at early ages (before age 15) and a slight decrease at later ages. Additive genetic coef- 
ficients of variations (CV) for four traits showed a decreasing trend with age and the decreasing rate was rapid at early ages and minor at later ages. Heritability for four traits was relatively stable with minor fluctuation. For across-age classes, heritability was the highest for height, intermediate for RYC, and lowest for volume and $\mathrm{DBH}$. RYC had highly positive genetic correlations with three growth characteristics. Genotype-by-environment interaction for four traits was stronger at Yunan than at other testing sites. Age-age genetic correlations were high for four traits studied, reaching 0.7 after age 9 for most analyses. Early selection at age 13 was highly effective for height, age 15 for $\mathrm{DBH}$ and volume, and age 11 for RYC.

Key words: Pinus massoniana, resin-yielding capacity, progeny trial, age-age correlation, early selection efficiency.

\section{Introduction}

Masson pine (Pinus massoniana Lamb) is a native species of pine growing in a wide area of central and southern China. It is an evergreen tree reaching $45 \mathrm{~m}$ in height and $1 \mathrm{~m}$ in diameter at breast height, with a broad, rounded crown of long branches (ZENG and YUE, 1984). Besides its wide uses in wood, pulp and paper industry, this species also has long been employed as a main source of resin, a hydrocarbon secretion of many plants widely used to produce resin and turpentine for chemical industry (TOMUSIAK and MAGNUszeWsKI, 2009; COPPEn and Hone, 1995). China is the leading producer of resin in the world (WANG et al., 2006). During the 1990 s, China annually exported a total of 200,000 tons of resin to more than 40 countries, accounting for about $50 \%$ of resin traded in the world (LIU, 2001). Of the pine resin resources, approximate $95 \%$ comes from masson pine (ZENG and YUE, 1984). However, due to its high commercial value, masson pine has been subjected to over-exploitation during past decades, leading to a gradual decrease in genetic resources (SHI and LI, 1998).

Genetic improvement of this species was initiated in late $1950 \mathrm{~s}$, and since then, active breeding programs have been launched for the purpose of improving the wood yield and quality (HoNG et al., 2010; LIN et al., 2010; ZHOU et al., 2000; LIAN et al., 2006. Genetic analyses have detected significant variation in growth and wood density among masson pine provenances and families. Estimates of heritability coefficients in progeny test have shown moderate genetic control for growth and wood density. Genetic gains have been estimated at $3.8 \%$ for height, $14.4 \%$ for $\mathrm{DBH}$, and $27.5 \%$ for real volume (Hong et al., 2010). These studies show high potential of this species for improving growth and wood quali- ty through selection of the more productive provenances and families.

In term of resin-yielding capacity (RYC) of this species, there are few breeding practices reported so far. In south China, the main resin-yielding region of this species, the annual planting area has reached approximately $45 \%$ of total plan (ZENG and YuE, 1984). However, the majority of seedlings used for afforestation have not been subjected to genetic improvement, and the pine plantations have limited contribution to the increment of RYC. The need for genetically improved seedlings has long been recognized. To date, tree improvement program concerning $\mathrm{RYC}$ of the species is still in its infancy and started with the selection of 182 plus-trees from natural stands in main resin-yielding region. Forward or backward selecting genetically superior individuals through progeny test trials has been considered a preferred method (KUMAR and LEE, 2002).

The objectives of this study were (1) to calculate the variance components and heritabilities for growth characteristics and RYC in three progeny trials, (2) to estimate the coefficients of age-age correlation and early selection efficiency.

\section{Material and Methods}

\section{Sampling and experimental design}

In 1982 intensive survey and inspection of natural stands of masson pine were conducted in six provinces of south China, including Guangdong, Guangxi, Anhui, Jiangxi, Fujian and Zhejiang. This resulted in the initial selection of 182 candidate plus trees along with 910 check trees. Each candidate was subsequently scored for traits of interest in relation to five selected check trees (LEDIG, 1974). The scored traits included RYC, height (HT), diameter at breast height $(\mathrm{DBH})$, volume of individual tree (VOL), stem form and crown size. Ninetythree trees were screened out of these 182 candidates from the first round checking procedure. Subsequently, these 93 trees were subject to a second round checking with RYC as the primary selection criteria in the next year and a total of 50 plus trees with high RYC were identified for the progeny testing (Table 1). The origin of these 50 plus trees was located in five prefectures of Guangdong province, in the range of latitude $21^{\circ} 55^{\prime} \mathrm{N}-23^{\circ} 87^{\prime} \mathrm{N}$, longitude $110^{\circ} 47^{\prime} \mathrm{E}-114^{\circ} 41^{\prime} \mathrm{E}$ and at elevation from $35 \mathrm{~m}$ to $458 \mathrm{~m}$ (Table 1 ).

Seeds from these 50 plus trees were collected for establishment of progeny trials in 1984 (Table 2). Openpollinated families from these trees were included in

Table 1. - General information of plus-trees of Masson pine used in progeny testing. GD represents Guangdong province.

\begin{tabular}{|c|c|c|c|c|c|}
\hline Origita & $\begin{array}{l}\text { I atitude } \\
\text { (N) }\end{array}$ & $\begin{array}{l}\text { I.ongitude } \\
\text { (E) }\end{array}$ & $\begin{array}{l}\text { Altitude } \\
\text { (metres) }\end{array}$ & $\begin{array}{l}\text { Antrual } \\
\text { (rnmiannum) }\end{array}$ & $\begin{array}{l}\text { No. of plus } \\
\text { Lees }\end{array}$ \\
\hline I leyuan, (iI) & $23^{\circ} 43^{\prime}$ & $114^{\circ} 41^{\prime}$ & $35-109$ & $18 \times 1.8$ & 2 \\
\hline Deqing, GD & $23 \circ 87^{\prime}$ & $110^{\circ} 47^{\prime}$ & $122-265$ & 1513.0 & 2 \\
\hline Gaozhou, GD & $21^{\circ 5}$ & $110^{\circ 5} 1^{\prime}$ & 104388 & 1892.7 & 10 \\
\hline Xinyi, GD & $22^{\circ} 22^{\prime}$ & $110^{2} 56^{\prime}$ & 150458 & 1481.7 & 22 \\
\hline Yunan, GD & $23^{\mathrm{c}} 15^{\prime}$ & $111^{\circ} 32^{\prime}$ & $127-339$ & 1433.0 & 14 \\
\hline
\end{tabular}


Table 2. - Details of trial locations of masson pine progeny testing.

\begin{tabular}{|c|c|c|c|}
\hline Pararneter & Xinlyj & Yunars & Heyuan \\
\hline Latitude & $22^{\circ} 25^{\prime} \mathrm{N}$ & $23^{\circ} 15^{\prime} \mathrm{V}$ & $23^{\circ} 45^{\prime} \mathrm{N}$ \\
\hline Longitude & $110^{\circ} 56^{\prime} \mathrm{F}$ & $111^{\circ} 32^{\prime} \mathrm{H}$ & $114^{\circ} 4 l^{\prime} \mathrm{F}$ \\
\hline Elevation ( $m$ ) & 450.0 & 100.0 & 41.1 \\
\hline Rainfall (mm) & 1800.0 & 1400.0 & 1665.0 \\
\hline Annual average temperature $\left({ }^{\circ} \mathrm{C}\right)$ & 22.3 & 21.3 & 21.1 \\
\hline
\end{tabular}

three trials, which were established at three locations: Xinyi, Yunan and Heyuan, in Guangdong province (Table 2). The trials used randomized complete block design with four-tree row plots and 10 replications per site. Seedlings were planted with $3 \times 3 \mathrm{~m}$ spacing. The trees in seven randomly selected replications were measured for RYC in grams per day, HT in meters and DBH in centimeters at ages $7,9,11,13,15,20$ (only for Xinyi), 24 (only for Heyuan) and 26 (only for Yunan) years after planting.

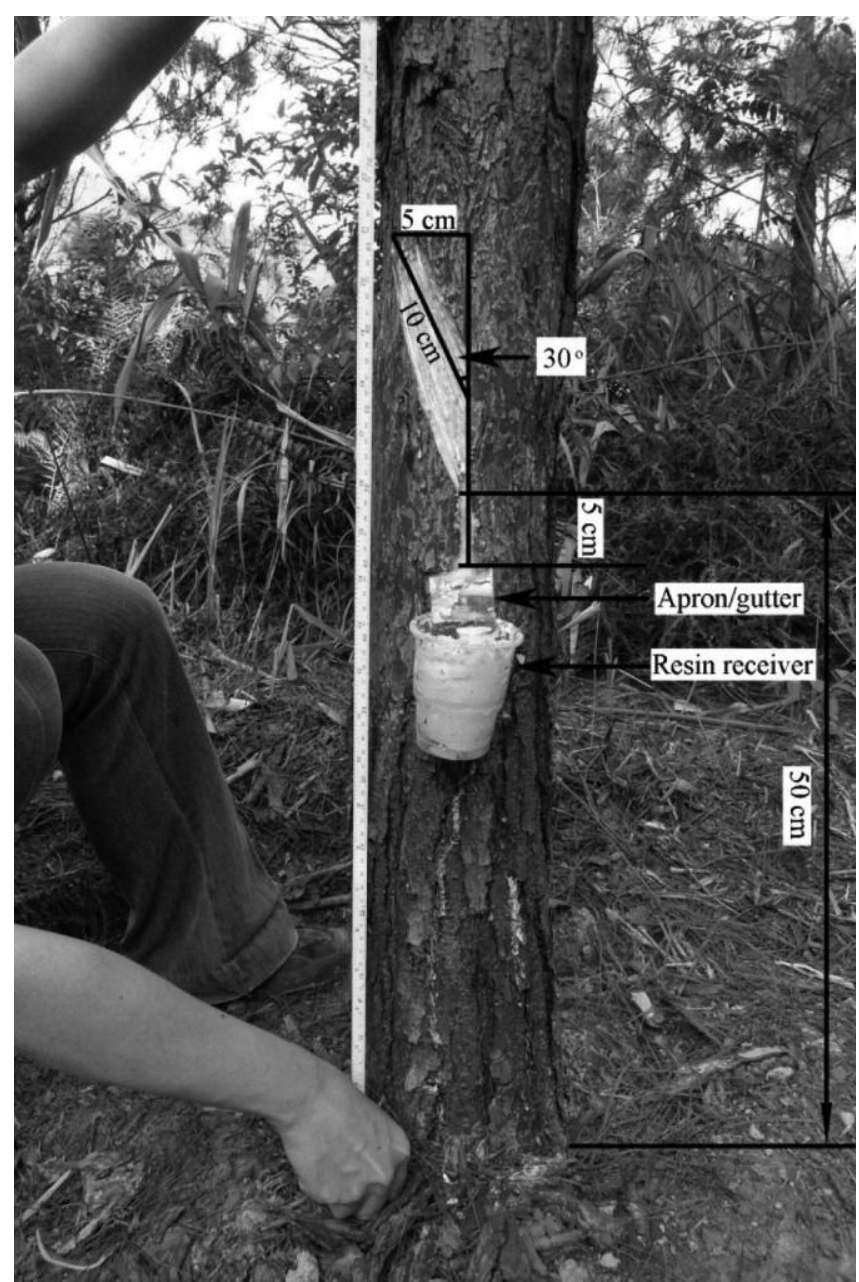

Figure 1. - Narrow face system of resin tapping. A total of 15 cuttings (once each day) are performed for resin tapping on each testing tree, leading to a tapping face of $5 \mathrm{~cm}$ wide and $10 \mathrm{~cm}$ long.
The resin tapping was carried out using the narrow face systems as described by CoPPEN and Hone (1995). In mid-August of each tapping year, the trees were cut once each day by a removal of a sliver of wood from the stem without the application of stimulant. In order to reduce damage to trees, the narrow tapping face was 5 cm wide and only 15 cuts were performed on the trees (Fig. 1).

HT and DBH were used to compute volume of individual tree outside bark per tree as described by ZENG and YUE (1984):

$$
\operatorname{VOL}\left(\mathrm{m}^{3}\right)=6.2341803 \times 10^{-5} \times \mathrm{DBH}^{1.8551497} \times \mathrm{HT}^{0.95682492}
$$

Where VOL is the volume of individual tree in cubic meters; $\mathrm{DBH}$ is the diameter at breast height in meters; and HT is the height of the tree in meters. An edition and cleaning was made to data prior to statistical analysis. Observations lying outside the normal range were eliminated to avoid inflation of error variances and misleading estimates. Deleted measurements accounted for less than $3 \%$ of the observations for all tests.

\section{Statistical Analysis}

Single-site analyses were conducted on each test site for the traits RYC, HT, DBH and VOL. Variance components were estimated using the SAS program, PROC GLM and VARCOMP (SAS Institute, 1999). The following model was used for analysis of variance (ANOVA) for each trait at each test site:

$$
Y_{i j k}=\mu+F_{i}+R_{j}+(F R)_{i j}+e_{k(i j)}
$$

where: $Y_{i j k}=$ the observation of the dependent variable of the $k$-th tree of the $i$-th family in $j$-th block (R)

$\mu=$ the overall mean

$F_{i}=$ the random effect of $i$-th family

$R_{j}=$ the fixed effect of $j$-th block

$(F R)_{i j}=$ the plot error

$e_{k(i j)}=$ the within-plot error.

The single-site family $\left(h^{2}{ }_{b f}\right)$ and individual $\left(h^{2}{ }_{b i}\right)$ heritabilities for each trait were calculated using the variance components and the following formulae:

$$
\begin{aligned}
& h_{b f}^{2}=\frac{\sigma_{f}^{2}}{\sigma_{f}^{2}+\sigma_{e}^{2} / n_{h} b+\sigma_{f b}^{2} / b} \\
& h_{b i}^{2}=\frac{4 \sigma_{f}^{2}}{\sigma_{f}^{2}+\sigma_{f b}^{2}+\sigma_{e}^{2}}
\end{aligned}
$$

where: 4 = the coefficient for estimating $h^{2}{ }_{b i}$ of half-sib families.

$n_{h}=$ harmonized average number of trees in each block

$b=$ block number

$\sigma_{f}^{2}=$ the family variance

$\sigma_{f b}^{2}=$ the family-by-block variance

$\sigma_{e}^{2}=$ the residual variance.

Resin-yielding capacity (RYC) was calculated as:

$\mathrm{RYC}=\frac{\mathrm{Wt}}{\mathrm{D} \cdot \mathrm{Wd} / \mathrm{C}}$ 
where: $\mathrm{Wt}=$ total weight of collected resin of a tree

$\mathrm{D}=$ the cutting times for resin tapping on each tree

$\mathrm{Wd}=$ the total width of narrow tapping face

$\mathrm{C}=$ the circumference of trunk where the bark was cut.

The following model was used for across-site analyses:

$$
Y_{i j k l}=\mu+S_{i}+F_{j}+R_{k(i)}+(S F)_{i j}+(F R)_{j k(i)}+e_{l(i j k)}
$$

where: $Y_{i j k l}=$ the observation of the dependent variable of the $l$-th tree of the $j$-th family $(F)$ in $k$-th block $(R)$ within the $i$-th site $(S)$

$\mu=$ the overall mean

$S_{i}=$ the $i$-th site effect $(S)$

$F_{j}=$ the random effect of $j$-th family $(F)$

$R_{k(i)}=$ the fixed effect of $k$-th block $(R)$

$(S F)_{i j}=$ the family-by-site $(\mathrm{G} \times \mathrm{E})$ interaction

$(F R)_{j k(i)}=$ the plot error

$e_{l(i j k)}=$ the within-plot error.

The across-site family $\left(h_{f}^{2}\right)$ and individual $h_{i}^{2}$ ) heritabilities for each trait were calculated using the variance components and the following formulae,

$$
\begin{aligned}
& h_{f}^{2}=\frac{\sigma_{f}^{2}}{\sigma_{e}^{2} / n_{h} b s+\sigma_{f b}^{2} / b s+\sigma_{f s}^{2} / s+\sigma_{f}^{2}} \\
& h_{i}^{2} \frac{4 \sigma_{f}^{2}}{\sigma_{e}^{2}+\sigma_{f b}^{2}+\sigma_{f s}^{2}+\sigma_{f}^{2}}
\end{aligned}
$$

where: $\sigma_{f}^{2}=$ the family variance

$n_{h}=$ the harmonized average number of trees in each block

$b=$ the block number in each site

$s=$ the site number

$\sigma_{f b}^{2}=$ the family-by-block variance

$\sigma_{f s}^{2}=$ the family-by-site variance

$\sigma_{e}^{2}=$ the residual variance.

The genetic correlation between performances of halfsib families for the same trait in different environments is termed a type B genetic correlation (BURDON, 1977; YAMADA, 1962). The magnitude of the genotype by environment interaction at the additive level was measured by using estimates of type B genetic correlation $\left(r_{B g}\right)$ following BURDON (1972).

$$
r_{B g}=\frac{\operatorname{cov}_{g_{x y}}}{\sigma_{g_{x}} \times \sigma_{g_{y}}}
$$

where: $r_{B g}=$ genetic correlation between one trait at environments $\mathrm{x}$ and $\mathrm{y}$

$\operatorname{cov}_{g_{x y}}=$ covariance for groups between the trait as it is expressed at environments $\mathrm{x}$ and $\mathrm{y}$

$\sigma_{g_{x}}$ and $\sigma_{g_{y}}$ are square roots of variances of group means at environments $\mathrm{x}$ and $\mathrm{y}$.

Juvenile- to mature-age genetic correlations $(r)$ were calculated as:

$$
r=\frac{\operatorname{cov}_{f J M}}{\sigma_{f J} \times \sigma_{f M}}
$$

where: $\operatorname{cov}_{f J M}=$ the family covariance among juvenile $(J)$ and mature age $(M) ; \sigma_{f J}$ and $\sigma_{f M}$ are square roots of family variances for juvenile $(J)$ and mature age $(M)$.

In this study, the last measurement year at each site was considered the mature age, namely age 20 at Xinyi, age 24 at Heyuan and age 26 at Yunan. The efficiency $\left(E f_{g e n}\right)$ of juvenile selection relative to mature age was calculated as:

$$
E f_{\text {gen }}=\frac{i_{J} r_{\mathrm{A}} h_{J}}{i_{M} h_{M}}
$$

where: $r_{A}=$ the additive genetic correlation between juvenile and mature ages; $i_{J}$ and $i_{M}$ are selection intensities at juvenile $(J)$ and mature age $(M) ; h_{J}$ and $h_{M}$ are square roots of the heritability at the juvenile $(J)$ and mature ages $(M)$; The same selection intensity for the juvenile and mature ages was used in this calculation (Wu et al., 2007; KUMAR and LEE, 2002).

\section{Results}

\section{Trend of annual trait means}

Annual survival rates of testing progenies at three sites were recorded and summarized in Table 3 . The survival rates decreased gradually with age. At the mature ages, $73 \%$ of trees remained at Xinyi (age 20), $63 \%$ at Heyuan (age 24) and 68\% at Yunan (age 24), respectively. Nevertheless, there was no significant variation in survival rate among families at each testing site (data not shown).

Overall means for HT, DBH, VOL (eq. 1) and RYC (eq. 5 ) were shown in Figure 2. The means of three growth traits increased gradually with age. The trees in Heyuan displayed the maximum early growth (before age 11) and low midterm growth from age 11 to 15 . The seedlings in Xinyi showed the maximum later growth (after age 11). The mean of individual volume increased from $0.018 \mathrm{~m}^{3}$ at age 7 to $0.181 \mathrm{~m} 3$ at mature age 20, suggesting that Xinyi were more suitable for growth of masson pine. In comparison, the trees in Yunan maintained low growth rate without dramatic fluctuation. The mean of individual volume was only $0.145 \mathrm{~m}^{3}$ at mature age 26.

In general, RYC showed a rapid increase at early ages and a subsequent slow decrease at mature ages. It displayed significant differences at three testing sites $(P<0.0001)$. Heyuan produced the highest yield of resin at early ages from 7 to 11 , varying from $22.95 \mathrm{~g} / \mathrm{d}$ to $44.83 \mathrm{~g} / \mathrm{d}$. Xinyi showed the maximum yield at later ages from 13 to 20, reaching the maximum $50.55 \mathrm{~g} / \mathrm{d}$ at age 15. While, Yunan always exhibited the lowest resin yield except at the beginning age 7 .

Table 3. - Annual survival rate of masson pine at three testing sites.

\begin{tabular}{ccccccccc}
\hline Testing & \multicolumn{8}{c}{ Age (years) } \\
\cline { 2 - 9 } sile & 7 & 9 & 11 & 13 & 15 & 20 & 24 & 26 \\
\hline Xinyi & 91.8 & 94.5 & 77.3 & 77.3 & 74.4 & 72.9 & - & - \\
Yunanl & 92.0 & 83.7 & 83.4 & 83.0 & 82.7 & & & 68.0 \\
Heyuan & 86.5 & 80.8 & 76.1 & 75.3 & 73.9 & - & 63.0 & - \\
\hline
\end{tabular}




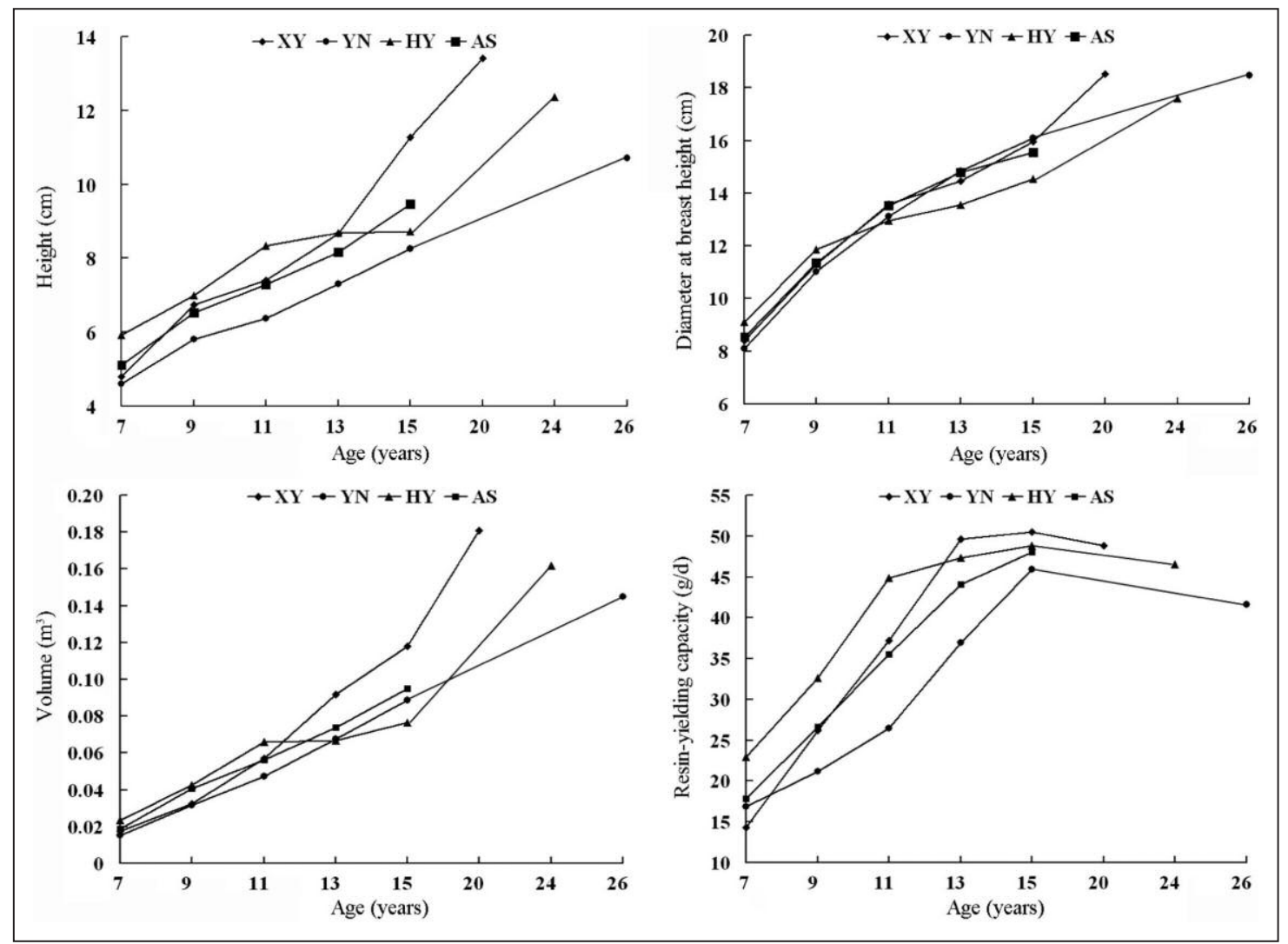

Figure 2. - Production trends of four traits from ages 7 to 26 at three testing sites. XY=Xinyi; YN=Yunan; $\mathrm{HY}=$ Heyuan; AS = Across-site. The same below.
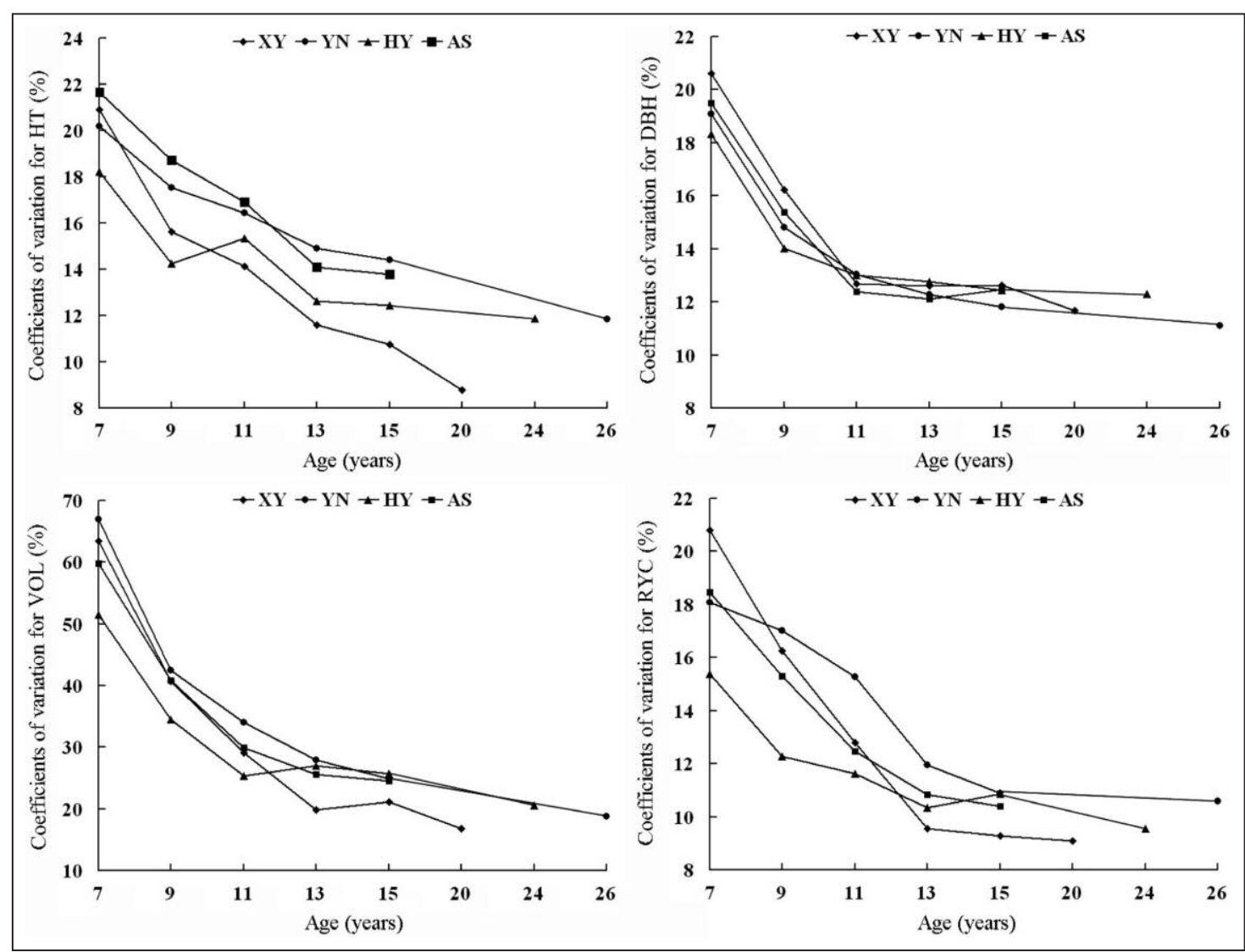

Figure 3. - Trends in coefficients of variation (CV; \%) for four traits from age 7 to 26 at three testing sites; $\mathrm{HT}=$ height; $\mathrm{DBH}=$ diameter at breast height; $\mathrm{VOL}=$ volume; $\mathrm{RYC}=$ resin-yielding capacity. The same below. 


\section{Analysis of variances}

For single-site analysis (eq. 2), family had significant $(P<0.05)$ effects on four traits at all ages. The effects of block and block-by-family interaction were also significant for majority of analyses. Similarly for across-site analysis (eq. 6), family had significant $(P<0.05)$ effects on four traits at all ages. Testing site also played a significant role $(P<0.05)$ in affecting four traits at all ages. The effects of block, site-by-family interaction, and block-by-family interaction were significant in most cases.

Table 4. - Heritability estimates for height from ages 7 to 26 at three testing sites.

\begin{tabular}{|c|c|c|c|c|c|c|c|c|}
\hline \multirow{2}{*}{$\begin{array}{c}\text { Age } \\
\text { (years) }\end{array}$} & \multicolumn{2}{|c|}{ Xinyi } & \multicolumn{2}{|c|}{ Yuntran } & \multicolumn{2}{|c|}{ l leyuan } & \multicolumn{2}{|c|}{ Across-site } \\
\hline & $h_{3 i}^{2}$ & $h_{b ;}^{2}$ & $h_{b i}^{2}$ & $h_{l: f}^{2}$ & $h_{b i}^{2}$ & $h_{l:}^{2}$ & $h_{i}^{2}$ & $h^{2}$ \\
\hline 7 & $\begin{array}{c}0.32 \\
(0.14)\end{array}$ & $\begin{array}{c}0.36 \\
(0.11)\end{array}$ & $\begin{array}{c}0.33 \\
(0.19)\end{array}$ & $\begin{array}{c}0.30 \\
(0.09)\end{array}$ & $\begin{array}{c}0.61 \\
(0.12)\end{array}$ & $\begin{array}{c}0.54 \\
(0.18)\end{array}$ & $\begin{array}{c}0.17 \\
(0.05)\end{array}$ & $\begin{array}{c}0.36 \\
(0.12)\end{array}$ \\
\hline 9 & $\begin{array}{c}0.45 \\
(0.12)\end{array}$ & $\begin{array}{c}0.38 \\
(0.08)\end{array}$ & $\begin{array}{c}0.31 \\
(0.09)\end{array}$ & $\begin{array}{c}0.34 \\
(0.15)\end{array}$ & $\begin{array}{c}0.59 \\
(0.05)\end{array}$ & $\begin{array}{c}0.55 \\
(0.14)\end{array}$ & $\begin{array}{c}0.22 \\
(0.07)\end{array}$ & $\begin{array}{c}0.54 \\
(0.11)\end{array}$ \\
\hline 11 & $\begin{array}{c}0.38 \\
(0.13)\end{array}$ & $\begin{array}{c}0.42 \\
(0.14)\end{array}$ & $\begin{array}{c}0.48 \\
(0.13)\end{array}$ & $\begin{array}{c}0.39 \\
(0.16)\end{array}$ & $\begin{array}{c}0.42 \\
(0.14)\end{array}$ & $\begin{array}{c}0.48 \\
(0.14)\end{array}$ & $\begin{array}{c}0.17 \\
(0.06)\end{array}$ & $\begin{array}{c}0.30 \\
(0.11)\end{array}$ \\
\hline 13 & $\begin{array}{c}0.40 \\
(0.14)\end{array}$ & $\begin{array}{c}0.43 \\
(0.04)\end{array}$ & $\begin{array}{c}0.49 \\
(0.16)\end{array}$ & $\begin{array}{c}0.51 \\
(0.04)\end{array}$ & $\begin{array}{c}0.41 \\
(0.09)\end{array}$ & $\begin{array}{c}0.40 \\
(0.11)\end{array}$ & $\begin{array}{c}0.13 \\
(0.07)\end{array}$ & $\begin{array}{c}0.36 \\
(0.08)\end{array}$ \\
\hline 15 & $\begin{array}{c}0.47 \\
(0.08)\end{array}$ & $\begin{array}{c}0.45 \\
(0.12)\end{array}$ & $\begin{array}{c}0.39 \\
(0.15)\end{array}$ & $\begin{array}{c}0.34 \\
(0.11)\end{array}$ & $\begin{array}{c}0.5) \\
(0.14)\end{array}$ & $\begin{array}{c}0.46 \\
(0.05)\end{array}$ & $\begin{array}{c}0.21 \\
(0.10)\end{array}$ & $\begin{array}{c}0.44 \\
(0.06)\end{array}$ \\
\hline 20 & $\begin{array}{c}0.40 \\
(0.11)\end{array}$ & $\begin{array}{c}0.43 \\
(0.07)\end{array}$ & - & - & - & - & - & - \\
\hline 24 & & & & & $\begin{array}{c}0.38 \\
(0.11)\end{array}$ & $\begin{array}{c}0.43 \\
(0.13)\end{array}$ & & \\
\hline 26 & - & - & $\begin{array}{c}0.39 \\
(0.11)\end{array}$ & $\begin{array}{c}0.45 \\
(0.15)\end{array}$ & - & - & - & - \\
\hline
\end{tabular}

Values in parentheses are approximate standard errors; $h_{b i}^{2}$ and $h_{b f}^{2}$ indicate individual and family heritability, respectively, from single-site analyses; $h_{i}^{2}$ and $h_{f}^{2}$ represent individual and family heritability, respectively, from across site analyses. Dashes indicate no estimates. The same below.

Table 5. - Heritability estimates for diameter at breast height from ages 7 to 26 at three testing sites.

\begin{tabular}{|c|c|c|c|c|c|c|c|c|}
\hline \multirow{2}{*}{$\begin{array}{c}\text { Age } \\
\text { (ycars) }\end{array}$} & \multicolumn{2}{|c|}{ Xinyi } & \multicolumn{2}{|c|}{ Yunnan } & \multicolumn{2}{|c|}{ I leyuan } & \multicolumn{2}{|c|}{ Across-site } \\
\hline & $h_{b i}^{2}$ & $h^{2}$ & $h_{h t}^{2}$ & $h_{h}^{2}$ & $h_{h,}^{2}$ & $h_{i i}^{2}$ & $h_{i}^{2}$ & $h_{f}^{2}$ \\
\hline 7 & $\begin{array}{c}0.14 \\
(0.05)\end{array}$ & $\begin{array}{c}0.20 \\
(0.12)\end{array}$ & $\begin{array}{c}0.20 \\
(0.09)\end{array}$ & $\begin{array}{c}0.27 \\
(0.14)\end{array}$ & $\begin{array}{c}0.29 \\
(0.08)\end{array}$ & $\begin{array}{c}0.38 \\
(0.10)\end{array}$ & $\begin{array}{c}0.27 \\
(0.12)\end{array}$ & $\begin{array}{r}0.55 \\
(0.05)\end{array}$ \\
\hline 9 & $\begin{array}{c}0.15 \\
(0.08)\end{array}$ & $\begin{array}{c}0.20 \\
(0.10)\end{array}$ & $\begin{array}{c}0.25 \\
(0.05)\end{array}$ & $\begin{array}{c}0.23 \\
(0.11)\end{array}$ & $\begin{array}{c}0.16 \\
(0.11)\end{array}$ & $\begin{array}{c}0.24 \\
(0.04)\end{array}$ & $\begin{array}{c}0.16 \\
(0.05)\end{array}$ & $\begin{array}{r}0.50 \\
(0.13)\end{array}$ \\
\hline 11 & $\begin{array}{c}0.13 \\
(0.10)\end{array}$ & $\begin{array}{c}0.20 \\
(0.09)\end{array}$ & $\begin{array}{c}0.11 \\
(0.04)\end{array}$ & $\begin{array}{c}0.15 \\
(0.09)\end{array}$ & $\begin{array}{c}0.13 \\
(0.07)\end{array}$ & $\begin{array}{c}0.23 \\
(0.12)\end{array}$ & $\begin{array}{c}0.10 \\
(0.04)\end{array}$ & $\begin{array}{r}0.45 \\
(0.02)\end{array}$ \\
\hline 13 & $\begin{array}{c}0.16 \\
(0.07)\end{array}$ & $\begin{array}{c}0.22 \\
(0.15)\end{array}$ & $\begin{array}{c}0.15 \\
(0.06)\end{array}$ & $\begin{array}{c}0.20 \\
(0.11)\end{array}$ & $\begin{array}{c}0.14 \\
(0.09)\end{array}$ & $\begin{array}{c}0.22 \\
(0.08)\end{array}$ & $\begin{array}{c}0.08 \\
(0.04)\end{array}$ & $\begin{array}{r}0.40 \\
(0.15)\end{array}$ \\
\hline 15 & $\begin{array}{c}0.17 \\
(0.07)\end{array}$ & $\begin{array}{c}0.24 \\
(0.13)\end{array}$ & $\begin{array}{c}0.29 \\
(0.14)\end{array}$ & $\begin{array}{c}0.37 \\
(0.10)\end{array}$ & $\begin{array}{c}0.24 \\
(0.04)\end{array}$ & $\begin{array}{c}0.34 \\
(0.14)\end{array}$ & $\begin{array}{c}0.23 \\
(0.12)\end{array}$ & $\begin{array}{r}0.60 \\
(0.15)\end{array}$ \\
\hline 20 & $\begin{array}{c}0.16 \\
(0.09)\end{array}$ & $\begin{array}{c}0.23 \\
(0.07)\end{array}$ & & & & & & \\
\hline 24 & - & - & - & - & $\begin{array}{c}0.22 \\
(0.14)\end{array}$ & $\begin{array}{c}0.30 \\
(0.14)\end{array}$ & - & - \\
\hline 26 & & & $\begin{array}{c}0.23 \\
(0.12)\end{array}$ & $\begin{array}{c}0.34 \\
(0.07)\end{array}$ & & & & \\
\hline
\end{tabular}

\section{Genetic coefficients of variations and heritability}

Trends in additive genetic coefficients of variations $(\mathrm{CV} ; \%)$ are presented in Figure 3. A decreasing trend of magnitude of $\mathrm{CV}$ was observed for four traits with age. The decreasing rate was rapid at early ages (before age 11) and minor at later ages (after age 11). In general, CV for overall VOL was much higher than that for overall $\mathrm{HT}, \mathrm{DBH}$ and RYC, and there was a larger scale of decline in the CV for VOL when compared with that for other traits.

In general, heritability for four traits was relatively stable (Tables 4 to 7 ). For single site analyses, there was no significant difference between family heritability (eq. 3 ) and individual heritability (eq. 4) for four traits. Heritability was the highest for height $(>0.30)$ at all ages,

Table 6. - Heritability estimates for individual volume from ages 7 to 26 at three testing sites.

\begin{tabular}{|c|c|c|c|c|c|c|c|c|}
\hline \multirow{2}{*}{$\begin{array}{c}\text { Age } \\
\text { (years) }\end{array}$} & \multicolumn{2}{|c|}{ Xinyi } & \multicolumn{2}{|c|}{ Yunnan } & \multicolumn{2}{|c|}{ I Ieyuan } & \multicolumn{2}{|c|}{ Across-site } \\
\hline & $h_{b i}^{2}$ & $h_{t}^{2}$ & $h_{b j}^{2}$ & $h_{i, t}^{2}$ & $h_{b i}^{2}$ & $h_{b i}^{2}$ & $h_{i}^{2}$ & $h_{j}^{2}$ \\
\hline 7 & $\begin{array}{c}0.18 \\
(0.06)\end{array}$ & $\begin{array}{c}0.17 \\
(0.04)\end{array}$ & $\begin{array}{c}0.26 \\
(0.10)\end{array}$ & $\begin{array}{c}0.27 \\
(0.14)\end{array}$ & $\begin{array}{c}0.21 \\
(0.05)\end{array}$ & $\begin{array}{c}0.30 \\
(0.15)\end{array}$ & $\begin{array}{c}0.16 \\
(0.05)\end{array}$ & $\begin{array}{c}0.43 \\
(0.13)\end{array}$ \\
\hline 9 & $\begin{array}{c}0.17 \\
(0,08)\end{array}$ & $\begin{array}{c}0.26 \\
(0.19)\end{array}$ & $\begin{array}{c}0.10 \\
(0.04)\end{array}$ & $\begin{array}{c}0.12 \\
(0.02)\end{array}$ & $\begin{array}{c}0.24 \\
(0.08)\end{array}$ & $\begin{array}{c}0.29 \\
(0.13)\end{array}$ & $\begin{array}{c}0.17 \\
(0.11)\end{array}$ & $\begin{array}{r}0.55 \\
(0.11)\end{array}$ \\
\hline 11 & $\begin{array}{c}0.16 \\
(0.10)\end{array}$ & $\begin{array}{c}0.28 \\
(0.08)\end{array}$ & $\begin{array}{c}0.16 \\
(0.07)\end{array}$ & $\begin{array}{c}0.14 \\
(0.05)\end{array}$ & $\begin{array}{c}0.28 \\
(0.12)\end{array}$ & $\begin{array}{c}0.37 \\
(0.10)\end{array}$ & $\begin{array}{c}0.15 \\
(0.11)\end{array}$ & $\begin{array}{c}0.51 \\
(0.05)\end{array}$ \\
\hline 13 & $\begin{array}{c}0.19 \\
(0.13)\end{array}$ & $\begin{array}{c}0.29 \\
(0.05)\end{array}$ & $\begin{array}{c}0.14 \\
(0.09)\end{array}$ & $\begin{array}{c}0.14 \\
(0.08)\end{array}$ & $\begin{array}{c}0.22 \\
(0.13)\end{array}$ & $\begin{array}{c}0.29 \\
(0.12)\end{array}$ & $\begin{array}{c}0.17 \\
(0.10)\end{array}$ & $\begin{array}{c}0.48 \\
(0.13)\end{array}$ \\
\hline 15 & $\begin{array}{c}0.14 \\
(0.06)\end{array}$ & $\begin{array}{c}0.24 \\
(0.11)\end{array}$ & $\begin{array}{c}0.32 \\
(0.13)\end{array}$ & $\begin{array}{c}0.35 \\
(0.10)\end{array}$ & $\begin{array}{c}0.34 \\
(0.14)\end{array}$ & $\begin{array}{c}0.40 \\
(0.05)\end{array}$ & $\begin{array}{c}0.19 \\
(0.07)\end{array}$ & $\begin{array}{c}0.53 \\
(0.12)\end{array}$ \\
\hline 20 & $\begin{array}{c}0.16 \\
(0.09)\end{array}$ & $\begin{array}{c}0.27 \\
(0.10)\end{array}$ & - & - & - & - & - & - \\
\hline 24 & - & - & - & - & $\begin{array}{c}0.48 \\
(0.03)\end{array}$ & $\begin{array}{c}0.42 \\
(0.16)\end{array}$ & - & - \\
\hline 26 & - & - & $\begin{array}{c}0.29 \\
(0.14)\end{array}$ & $\begin{array}{c}0.40 \\
(0.15)\end{array}$ & - & - & - & - \\
\hline
\end{tabular}

Table 7. - Heritability estimates for resin-yielding capacity from ages 7 to 26 at three testing sites.

\begin{tabular}{|c|c|c|c|c|c|c|c|c|}
\hline \multirow{2}{*}{$\begin{array}{c}\text { Age } \\
\text { (years) }\end{array}$} & \multicolumn{2}{|c|}{ Xinyi } & \multicolumn{2}{|c|}{ Yunnan } & \multicolumn{2}{|c|}{ Heyuan } & \multicolumn{2}{|c|}{ Across-site } \\
\hline & $h_{\partial j}^{2}$ & $h_{l l}^{2}$ & $h_{f i}^{2}$ & $h_{h, r}^{?}$ & $h_{b i}^{2}$ & $h_{i, i}^{2}$ & $h_{i}^{2}$ & $h_{i}^{2}$ \\
\hline 7 & $\begin{array}{c}0.23 \\
(0.11)\end{array}$ & $\begin{array}{c}0.35 \\
(0.10)\end{array}$ & $\begin{array}{c}0.28 \\
(0.02)\end{array}$ & $\begin{array}{c}0.36 \\
(0.10)\end{array}$ & $\begin{array}{c}0.11 \\
(0.09)\end{array}$ & $\begin{array}{c}0.18 \\
(0.10)\end{array}$ & $\begin{array}{c}0.17 \\
(0.08)\end{array}$ & $\begin{array}{c}0.58 \\
(0.11)\end{array}$ \\
\hline 9 & $\begin{array}{c}0.24 \\
(0.06)\end{array}$ & $\begin{array}{c}0.33 \\
(0.11)\end{array}$ & $\begin{array}{c}0.26 \\
(0.06)\end{array}$ & $\begin{array}{c}0.31 \\
(0.12)\end{array}$ & $\begin{array}{c}0.23 \\
(0.06)\end{array}$ & $\begin{array}{c}0.35 \\
(0.17)\end{array}$ & $\begin{array}{c}0.20 \\
(0.09)\end{array}$ & $\begin{array}{c}0.55 \\
(0.12)\end{array}$ \\
\hline 11 & $\begin{array}{c}0.13 \\
(0.08)\end{array}$ & $\begin{array}{c}0.22 \\
(0.14)\end{array}$ & $\begin{array}{c}0.25 \\
(0.07)\end{array}$ & $\begin{array}{c}0.29 \\
(0.07)\end{array}$ & $\begin{array}{c}0.14 \\
(0.05)\end{array}$ & $\begin{array}{c}0.22 \\
(0.06)\end{array}$ & $\begin{array}{c}0.13 \\
(0.05)\end{array}$ & $\begin{array}{c}0.52 \\
(0.05)\end{array}$ \\
\hline 13 & $\begin{array}{c}0.15 \\
(0.09)\end{array}$ & $\begin{array}{c}0.22 \\
(0.11)\end{array}$ & $\begin{array}{c}0.21 \\
(0.07)\end{array}$ & $\begin{array}{c}0.28 \\
(0.16)\end{array}$ & $\begin{array}{c}0.16 \\
(0.04)\end{array}$ & $\begin{array}{c}0.23 \\
(0.14)\end{array}$ & $\begin{array}{c}0.18 \\
(0.03)\end{array}$ & $\begin{array}{c}0.59 \\
(0.13)\end{array}$ \\
\hline 15 & $\begin{array}{c}0.15 \\
(0.08)\end{array}$ & $\begin{array}{c}0.22 \\
(0.15)\end{array}$ & $\begin{array}{c}0.25 \\
(0.12)\end{array}$ & $\begin{array}{c}0.33 \\
(0.15)\end{array}$ & $\begin{array}{c}0.30 \\
(0.11)\end{array}$ & $\begin{array}{c}0.40 \\
(0.16)\end{array}$ & $\begin{array}{c}0.18 \\
(0.09)\end{array}$ & $\begin{array}{c}0.54 \\
(0.12)\end{array}$ \\
\hline 20 & $\begin{array}{c}0.17 \\
(0.05)\end{array}$ & $\begin{array}{c}0.21 \\
(0.08)\end{array}$ & - & - & - & - & - & - \\
\hline 24 & - & - & - & - & $\begin{array}{c}0.31 \\
(0.17)\end{array}$ & $\begin{array}{c}0.39 \\
(0.10)\end{array}$ & - & - \\
\hline 26 & & & $\begin{array}{c}0.22 \\
(0.13)\end{array}$ & $\begin{array}{c}0.33 \\
(0.08)\end{array}$ & & & & \\
\hline
\end{tabular}


intermediate for RYC, and the lowest for DBH and VOL. With regard to across-site analyses, the family heritability (eq. 7) for four traits was much higher than individual heritability (eq. 8). At most ages, individual heritability for four traits was between 0.10 and 0.20 , and family heritability between 0.40 and 0.60 . The family heritability for RYC displayed the highest stability, ranging from 0.51 to 0.59 across ages. The family heritability for height varied from 0.30 to 0.54 , which was lower than that for other three traits at most ages.

\section{Genetic correlations among traits}

To identify the sites contributing to the significant site-by-family interaction, type B genetic correlations (eq. 9) between testing sites were estimated (Table 8). HT, DBH and VOL showed significant genetic correlations between Xinyi and Heyuan at most cases except for VOL at age 13. However, these growth traits displayed relatively low correlations between Xinyi and Yunan as well as between Heyuan and Yunan in majority of cases except for DBH between Heyuan and Yunan at ages 13 and 15, suggesting the existence of strong site-by-family interactions between these testing pairs. Additionally, no obvious trend of correlation was observed for each growth trait. With regard to RYC, significant correlations between Xinyi and Heyuan were detected between ages 9 to 15 . In contrast, the significant correlations were only observed between Heyuan and Yunan at ages 13 and 15 and no significant correlation was identified between Xinyi and Yunan. Interestingly, there was a gradual increase in correlations with age between Heyuan and Yunan, but not in other testing pairs.

To determine the interrelationships between RYC and other three growth traits, the genetic correlations were estimated (Table 9). Except for HT at age 7, significantly positive genetic correlations between RYC and growth traits were observed at three testing sites, but no obvious age trend was detected in the magnitude of these correlations. For majority of analyses, the correlations were between 0.50 and 0.85 . The correlations between pairs of traits did not differ apparently, nor did those

Table 8. - Estimates of type B genetic correlations and associated standard errors (in parenthesis) between testing sites for four traits.

\begin{tabular}{|c|c|c|c|c|c|c|c|c|c|c|c|c|}
\hline \multirow{2}{*}{$\begin{array}{l}\text { Age } \\
\text { (years) }\end{array}$} & \multicolumn{3}{|c|}{ HT } & \multicolumn{3}{|c|}{$\overline{\mathrm{DBH}}$} & \multicolumn{3}{|c|}{ VOL } & \multicolumn{3}{|c|}{$\mathrm{RYC}$} \\
\hline & $\mathrm{XY} / \mathrm{HY}$ & $\mathrm{XY} / \mathrm{YN}$ & $\mathrm{HY} / \mathrm{YN}$ & $\mathrm{XY} / \mathrm{HY}$ & $\mathrm{XY} / \mathrm{YN}$ & $\mathrm{HY} / \mathrm{YN}^{\circ}$ & $\mathrm{XY} / \mathrm{HY}$ & $\mathrm{XY} / \mathrm{YN}$ & $\mathrm{HY} / \mathrm{YN}$ & $\mathrm{XY} / \mathrm{HY}$ & $\mathrm{XY} / \mathrm{YN}$ & $\mathrm{HY} / \mathrm{YN}$ \\
\hline 7 & $\begin{array}{l}0.72^{\text {* }} \\
(0.10)\end{array}$ & $\begin{array}{c}0.03 \times \mathrm{S} \\
(0.03)\end{array}$ & $\begin{array}{c}0.18 \mathrm{NS} \\
(0.11)\end{array}$ & $\begin{array}{l}0.86^{* *} \\
(0.13)\end{array}$ & $\begin{array}{c}0.04 \mathrm{NS} \\
(0.03)\end{array}$ & $\begin{array}{c}0.25 \mathrm{NS} \\
(0.11)\end{array}$ & $\begin{array}{l}0.45^{*} \\
(0.14)\end{array}$ & $\begin{array}{c}0.11 \mathrm{NS} \\
(0.06)\end{array}$ & $\begin{array}{c}0.22 \mathrm{VS} \\
(0.08)\end{array}$ & $\begin{array}{c}0.33 \mathrm{NS} \\
(0.20)\end{array}$ & $\begin{array}{c}0.20 \mathrm{NS} \\
(0.08)\end{array}$ & $\begin{array}{c}0.21 \mathrm{VS} \\
(0.10)\end{array}$ \\
\hline 9 & $\begin{array}{l}0.92^{* *} \\
(0.11)\end{array}$ & $\begin{array}{c}0.09 \times \mathrm{S} \\
(0.06)\end{array}$ & $\begin{array}{c}0.05 \mathrm{NS} \\
(0.05)\end{array}$ & $\begin{array}{l}0.86^{* *} \\
(0.09)\end{array}$ & $\begin{array}{c}0.05 \mathrm{NS} \\
(0.06)\end{array}$ & $\begin{array}{c}0.22 \mathrm{NS} \\
(0.10)\end{array}$ & $\begin{array}{l}0.81^{* *} \\
(0.11)\end{array}$ & $\begin{array}{c}0.11 \mathrm{NS} \\
(0.10)\end{array}$ & $\begin{array}{c}0.27 \mathrm{VS} \\
(0.02)\end{array}$ & $\begin{array}{c}0.50^{*} \\
(0.14)\end{array}$ & $\begin{array}{c}0.23 \mathrm{NS} \\
(0.11)\end{array}$ & $\begin{array}{c}0.27 \text {. NS } \\
(0.05)\end{array}$ \\
\hline 11 & $\begin{array}{l}0.48^{*} \\
(0.15)\end{array}$ & $\begin{array}{c}0.13 \wedge \mathrm{S} \\
(0.09)\end{array}$ & $\begin{array}{c}0.15 \mathrm{NS} \\
(0.12)\end{array}$ & $\begin{array}{c}0.23 \mathrm{NS} \\
(0.12)\end{array}$ & $\begin{array}{c}0.09 \mathrm{NS} \\
(0.02)\end{array}$ & $\begin{array}{c}0.16 \mathrm{NS} \\
(0.10)\end{array}$ & $\begin{array}{l}0.40^{*} \\
(0.14)\end{array}$ & $\begin{array}{c}0.00 \mathrm{NS} \\
(0.00)\end{array}$ & $\begin{array}{c}0.25 \mathrm{VS} \\
(0.02)\end{array}$ & $\begin{array}{l}0.40^{*} \\
(0.14)\end{array}$ & $\begin{array}{c}0.31 \mathrm{NS} \\
(0.06)\end{array}$ & $\begin{array}{c}0.29 \mathrm{VS} \\
(0.02)\end{array}$ \\
\hline 13 & $\begin{array}{l}0.72^{*} * \\
(0.14)\end{array}$ & $\begin{array}{c}0.09 \mathrm{NS} \\
(0.01)\end{array}$ & $\begin{array}{c}0.20 \mathrm{NS} \\
(0.11)\end{array}$ & $\begin{array}{l}0.42^{*} \\
(0.03)\end{array}$ & $\begin{array}{c}0.01 \mathrm{NS} \\
(0.02)\end{array}$ & $\begin{array}{l}0.40^{*} * \\
(0.15)\end{array}$ & $\begin{array}{c}0.31 \wedge \mathrm{S} \\
(0.10)\end{array}$ & $\begin{array}{c}0.01 \mathrm{VS} \\
(0.01)\end{array}$ & $\begin{array}{c}0.31 \mathrm{VS} \\
(0.10)\end{array}$ & $\begin{array}{l}0.39^{*} \\
(0.15)\end{array}$ & $\begin{array}{c}0.24 \mathrm{NS} \\
(0.06)\end{array}$ & $\begin{array}{l}0.45 \% \\
(0.20)\end{array}$ \\
\hline 15 & $\begin{array}{l}0.73^{* *} \\
(0.05)\end{array}$ & $\begin{array}{c}0.07 \wedge \mathrm{S} \\
(0.04)\end{array}$ & $\begin{array}{c}0.14 \mathrm{NS} \\
(0.09)\end{array}$ & $\begin{array}{l}0.60^{* *} \\
(0.08)\end{array}$ & $\begin{array}{c}0.29 \mathrm{NS} \\
(0.17)\end{array}$ & $\begin{array}{l}0.42 * * * \\
(0.22)\end{array}$ & $\begin{array}{l}0.57^{* *} \\
(0.08)\end{array}$ & $\begin{array}{c}0.31 \mathrm{VS} \\
(0.10)\end{array}$ & $\begin{array}{c}0.26 \mathrm{VS} \\
(0.07)\end{array}$ & $\begin{array}{l}0.47^{*} \\
(0.20)\end{array}$ & $\begin{array}{c}0.28 \mathrm{NS} \\
(0.05)\end{array}$ & $\begin{array}{l}0.46^{*} \\
(0.14)\end{array}$ \\
\hline
\end{tabular}

NS: not statistically significant; * significant at $P<0.05$; ${ }^{* *}$ significant at $P<0.01$. HT: height; DBH: diameter at breast height; VOL: individual volume; $\mathrm{XY}=\mathrm{Xinyi}$ YN = Yunan; HY = Heyuan. The same below.

Table 9. - Estimates of age-age genetic correlations and associated standard errors (in parenthesis) between resin-yielding capacity and three growth traits.

\begin{tabular}{|c|c|c|c|c|c|c|c|c|c|}
\hline \multirow{2}{*}{$\begin{array}{c}\text { Age } \\
\text { (years) }\end{array}$} & \multicolumn{3}{|c|}{$\mathrm{HT}$} & \multicolumn{3}{|c|}{$\mathrm{DBH}$} & \multicolumn{3}{|c|}{ VOL } \\
\hline & Xinyi & Yunan & Heyuan & Xinyi & Yunan & Heyuan & Xinyi & Yunan & Heyuan \\
\hline 7 & $\begin{array}{c}0.36 \mathrm{NS} \\
(0.15)\end{array}$ & $\begin{array}{l}0.72 * * \\
(0.20)\end{array}$ & $\begin{array}{l}0.56^{* * *} \\
(0.22)\end{array}$ & $\begin{array}{l}0.76^{* * *} \\
(0.14)\end{array}$ & $\begin{array}{l}0.76^{* *} \\
(0.22)\end{array}$ & $\begin{array}{l}0.51 * \\
(0.13)\end{array}$ & $\begin{array}{l}0.81 * * \\
(0.10)\end{array}$ & $\begin{array}{l}0.75^{* *} \\
(0.06)\end{array}$ & $\begin{array}{l}0.67^{* *} \\
(0.11)\end{array}$ \\
\hline 9 & $\begin{array}{l}0.86^{* *} \\
(0.16)\end{array}$ & $\begin{array}{l}0.75 * * \\
(0.20)\end{array}$ & $\begin{array}{c}0.65^{* *} \\
(0.24)\end{array}$ & $\begin{array}{l}0.87^{* *} \\
(0.10)\end{array}$ & $\begin{array}{l}0.72^{* * *} \\
(0.23)\end{array}$ & $\begin{array}{l}0.51^{*} \\
(0.30)\end{array}$ & $\begin{array}{l}0.67 * * \\
(0.05)\end{array}$ & $\begin{array}{r}0.79 * * \\
(0.24)\end{array}$ & $\begin{array}{l}0.57^{* *} \\
(0.13)\end{array}$ \\
\hline 11 & $\begin{array}{l}0.51^{* *} \\
(0.06)\end{array}$ & $\begin{array}{l}0.73 * * \\
(0.11)\end{array}$ & $\begin{array}{l}0.59^{* *} \\
(0.23)\end{array}$ & $\begin{array}{l}0.71 * * \\
(0.18)\end{array}$ & $\begin{array}{l}0.64^{* *} \\
(0.24)\end{array}$ & $\begin{array}{c}0.66^{* *} \\
(0.11)\end{array}$ & $\begin{array}{l}0.80 * * \\
(0.15)\end{array}$ & $\begin{array}{r}0.66^{* *} \\
(0.15)\end{array}$ & $\begin{array}{c}0.83 * * \\
(0.11)\end{array}$ \\
\hline 13 & $\begin{array}{l}0.79 * * \\
(0.18)\end{array}$ & $\begin{array}{l}0.46^{*} \\
(0.17)\end{array}$ & $\begin{array}{l}0.42^{*} \\
(0.01)\end{array}$ & $\begin{array}{l}0.57 * * \\
(0.28)\end{array}$ & $\begin{array}{l}0.75^{* *} \\
(0.15)\end{array}$ & $\begin{array}{l}0.60^{* * *} \\
(0.22)\end{array}$ & $\begin{array}{l}0.63 * * \\
(0.15)\end{array}$ & $\begin{array}{c}0.74^{* *} \\
(0.15)\end{array}$ & $\begin{array}{l}0.54 * * \\
(0.25)\end{array}$ \\
\hline 15 & $\begin{array}{l}0.68^{* * *} \\
(0.10)\end{array}$ & $\begin{array}{l}0.66^{* * *} \\
(0.08)\end{array}$ & $\begin{array}{l}0.69^{* *} \\
(0.14)\end{array}$ & $\begin{array}{l}0.95^{* * *} \\
(0.05)\end{array}$ & $\begin{array}{l}0.60^{* * *} \\
(0.22)\end{array}$ & $\begin{array}{l}0.58^{* *} \\
(0.26)\end{array}$ & $\begin{array}{l}0.97 * * \\
(0.04)\end{array}$ & $\begin{array}{l}0.64^{* *} \\
(0.23)\end{array}$ & $\begin{array}{c}0.69^{* *} \\
(0.11)\end{array}$ \\
\hline 20 & $\begin{array}{l}0.60^{* * *} \\
(0.21)\end{array}$ & - & - & $\begin{array}{l}0.68 * \% \\
(0.20)\end{array}$ & - & - & $\begin{array}{l}0.63 * * \\
(0.14)\end{array}$ & - & - \\
\hline 24 & - & - & $\begin{array}{l}0.80^{* *} \\
(0.14)\end{array}$ & - & - & $\begin{array}{l}0.67^{* *} \\
(0.20)\end{array}$ & - & - & $\begin{array}{c}0.81 * * \\
(0.11)\end{array}$ \\
\hline 26 & - & $\begin{array}{l}0.54^{* * *} \\
(0.11)\end{array}$ & - & - & $\begin{array}{l}0.54^{*} \text { 水 } \\
(0.14)\end{array}$ & - & - & $\begin{array}{c}0.52^{* *} \\
(0.08)\end{array}$ & - \\
\hline
\end{tabular}


between testing sites. Nevertheless, RYC had the lowest correlation (0.355) with HT at Xinyi at age 7 and highest correlation (0.967) with VOL at Xinyi at age 15.

\section{Age-age correlations between earlier ages and mature} ages

The additive age-age genetic correlations (eq. 10) between earlier ages and mature age were shown in Figure 4 . There was an increasing trend in the magnitude of genetic correlations for HT at three testing sites, and the increase range at Xinyi and Yunan was much higher than that at Heyuan, particularly at early ages (ages 7 to 15$)$. The correlations for $\mathrm{DBH}$ at Heyuan were comparatively stable, and increased from 0.82 at age 7 to unity (1.0) at age 15. An analysis of correlations at early ages (ages 7 to 11) found a sharp increase at Xinyi and an obvious decrease at Yunan. Correlations at Yunan were much lower than those at Xinyi and Heyuan between ages 11 and 15 . The age trend of genetic correlations for VOL was highly similar with that for DBH. An increasing trend was observed at Xinyi and Heyuan, and the increase range at Xinyi was much higher than that at Heyuan. Correlations at Yunan displayed a sharp decrease at early ages (ages 7 to 9) and strong increase between ages 11 and 20. The correlations for RYC at Heyuan and Yunan were relatively stable at all ages and much higher $(>0.9)$ than those at Xinyi, particularly at early ages (ages 7 to 11). Correlations at Xinyi had a sharp increase between ages 9 and 13 .
Efficiency of early selection relative to selection at mature age

Efficiency of early selection (eq. 10) at Heyuan for HT was very high and stable $(>0.85)$ at all ages, and slowly increased from 0.87 at age 7 to 0.93 at age 15. Additionally, efficiency at Heyuan was much higher than that at Xinyi and Yunan at early ages (ages 7 to 11, Fig. 5). Efficiency at Xinyi and Yunan displayed a sharp increase at early ages. Early selection at age 15 almost had the same efficiency as selection at mature age for three testing sites. Similar to selection for HT, early selection for $\mathrm{DBH}$ at Heyuan was also very high (about 0.80 ) and stable at all ages (Fig. 5). Efficiency at Yunan was 0.77 at age 7 , but sharply decreased to middle level (0.550) at age 9 and maintained the middle level $(0.55 \sim 0.60)$ with minor increase from age 9 to 13 . At Xinyi, efficiency at age 7 was only 0.45 , but sharply increased to 0.69 at age 9 and 0.80 at age 11. Early selection at all ages at Heyuan, age 11 at Xinyi and age 15 at Yunan was almost as efficient as selection at mature age. The trend of selection efficiency for VOL was very similar with that for DBH (Fig. 5). At Yunan, there were an initial sharp decrease from age 7 to 9 and a subsequent dramatic increase from age 13 to 15 , leading to the fact that efficiency was much lower than that at Xinyi and Heyuan between age 9 and 13. At Heyuan, efficiency stably increased from 0.66 at age 7 to unity (1.0) at age 15. Early selection at age 9 at Xinyi, age 11 at Heyuan and age 15 at Yunan was almost as efficient as selection

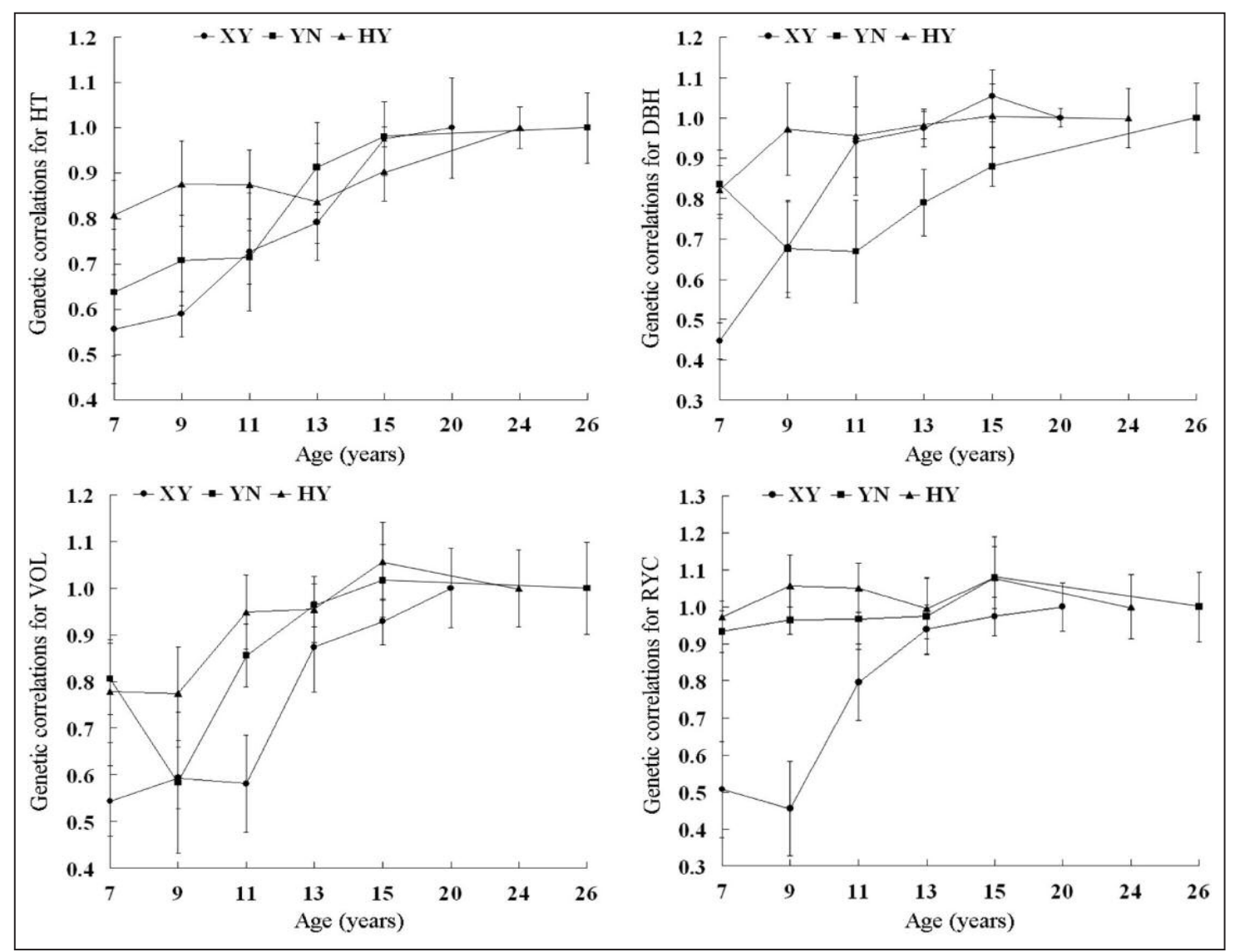

Figure 4. - Trends in age-age genetic correlations for four traits at three testing sites. The mature ages were $20(\mathrm{XY}), 24$ (HY) and 26 (YN), respectively; the earlier ages were from 7 to 15 . The same below. 


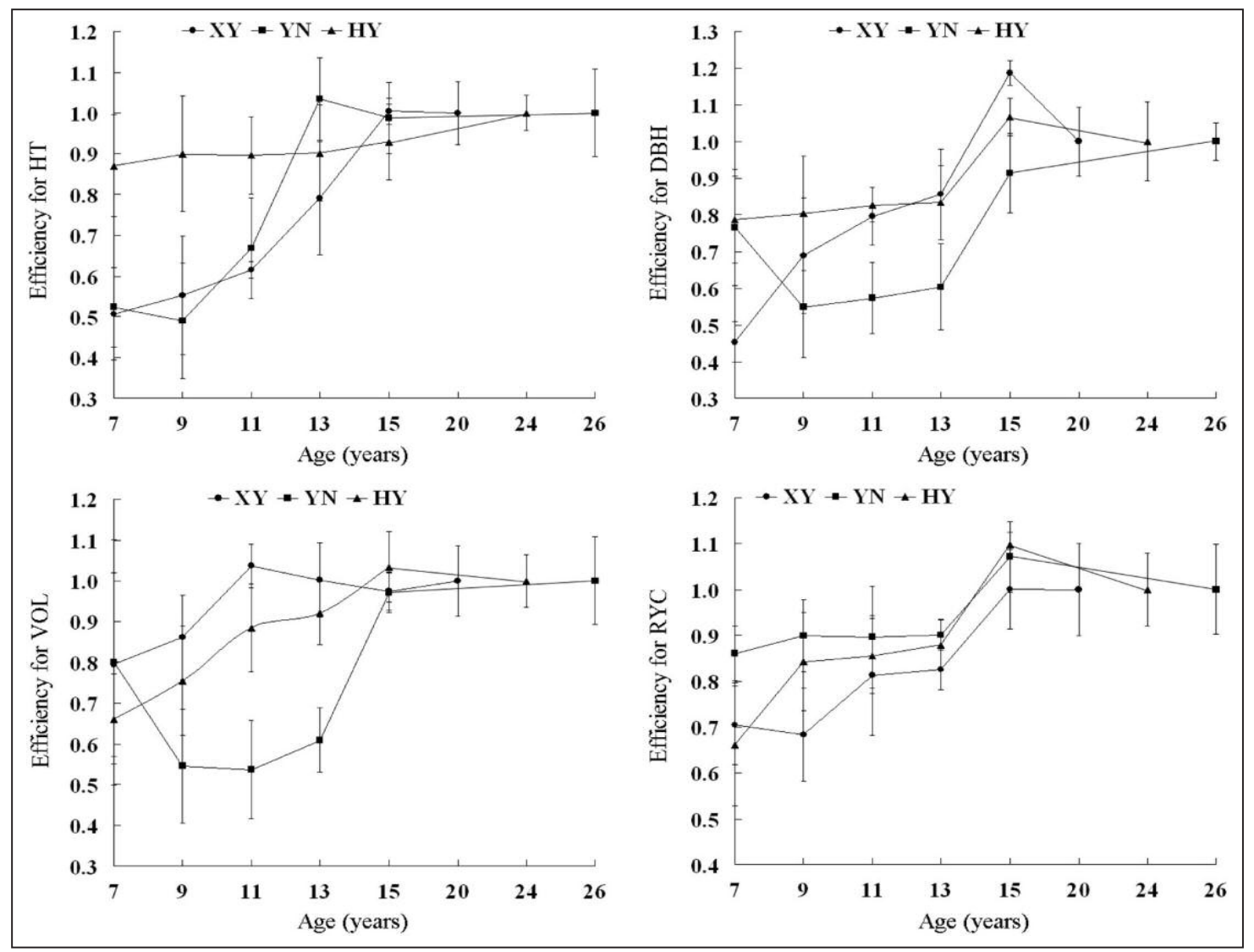

Figure 5. - Efficiency of early selection for mature age four traits at three testing sites.

at mature age. Efficiency for RYC at three testing sites displayed similar age trend. But efficiency at Yunan was stable and much higher than that at Xinyi and Heyuan at early ages (ages 7 to 13). Selection at all ages at Yunan, age 9 at Heyuan and age 11 at Xinyi had an efficiency $>0.8$.

\section{Discussion}

Since our ultimate goal is to improve resin-yielding capacity of masson pine, RYC was considered the primary selection criteria during the entire investigation of candidate plus trees in the natural forests of six provinces. Furthermore, two rounds of resin tapping was conducted for the purpose of screening for candidates with stable and high RYC. Therefore, it was reasonable for the fact that only 50 out of $182(27.5 \%)$ candidates were identified to be plus trees. Further analysis found that all of these 50 plus trees were distributed in Guangdong province, the principle region for resin production. Interestingly, our previous study on 274 masson pine trees from 6 provinces identified that RYC increased with the decrease of latitude (HE et al., 1999). Of these 6 provinces, Guangdong is located in the southeast part. This might imply geographic variation play a role in determining the RYC of masson pine. Similar geographic variation was also found in alder, in which provenances ranked differently at different sites and red alder provenances tended to perform better in both growth and survival at the sites near their origin (XIE, 2008).

We observed that family effect was significant for all growth traits and RYC in this study. This may not be surprising considering that family was detected having strong influence on growth traits of masson pine (HoNG et al., 2010; JIN et al., 2008a, 2008b; ZHOU et al., 2008; ZHANG et al., 2010). Since there were highly positive genetic correlation between RYC and growth characteristics in this study (Table 8), it is possible to detect the significant effect of family on RYC.

Similarly, site had significant effects on growth characteristics and RYC, suggesting important implications for seed transfer guidelines (XIE, 2008). Such effects might be attributed to differences in environmental conditions. A principal component analysis indicated that elevation, precipitation and evaporation were the three determinative factors for growth of masson pine $(\mathrm{XU}$, 1994). The central Guangdong province where there are numerous low mountains and hills with a middle elevation and high rainfall is considered the highly adaptable region for masson pine ( $\mathrm{XU}, 1994)$. Therefore it is not surprising to detect significant site-by-family interactions for three growth traits in this study.

Estimates of type B genetic correlations showed that Xinyi was well correlated with Heyuan and that Yunan was poorly correlated with other two sites (Table 8). It seemed that Yunan might be the site con- 
tributing to the strong site-by-family interaction. In terms of RYC, it was reported that the rise of temperature and humidity led to an increase in RYC, suggesting a close association between RYC and these two factors (ZHANG, 2001). In this study, Xinyi had significant type B correlations with Heyuan and relatively low (not significant) correlations with Yunan, which was in agreement with the RYC order of three testing sites at later ages: Xinyi $>$ Heyuan $>$ Yunan (Fig. 2). This result might be attributable to the fact that Xinyi had the highest elevation $(450 \mathrm{~m})$, rainfall $(1,800 \mathrm{~mm})$ and annual average temperature $\left(22.3^{\circ} \mathrm{C}\right)$, which is favorable for the growth and resin-yielding of masson pine (Table 2). Heyuan is closer to Xinyi and much higher than Yunan in RYC production (Fig. 2). It appears that RYC production of masson pine is more sensitive to rainfall when the trees are on low elevation with similar temperature (Table 2).

Estimation of CV suggested that there were plenty of genetic variations in four traits, particularly at early ages. The variation among masson pine families indicated the potential benefits of genetic improvement (HONG et al., 2010). However, further analysis found that CV for four traits displayed a decreasing trend with age. The decrease was found to be sharp at early ages and minor at later ages (Fig. 3). These trends were similar to wood density found in radiata pine (KUMAR and LEE, 2002), Norway spruce (Hylen, 1999) and Scots pine (FRIES and ERICSson, 2009). This might be reasonable considering that the survival rate of masson pines had a similar decreasing trend at three testing sites (Table 3). As trees mature, some tree may die while others may be suppressed by bigger neighboring trees due to inter-tree competition (WU et al., 2007). Additionally, there was a larger CV for volume of individual tree, which was in accordance with the results from other studies on masson pine (Hong et al., 2010; Du et al., 2010; Ji et al., 2005). Similar trends were also found in Pinus caribaea Mor. var. bahamensis (SEBBENN et al., 2008) and radiata pine (KUMAR and LEE, 2002). This may be explained by the fact that the volume was calculated by multiplying height and DBH.

Heritability for each trait was relatively stable at all ages at three sites in this study. This is similar with the results of radiata pine in which heritability for $\mathrm{DBH}$ increased to a stable value at age 6 ( $\mathrm{LI}$ and $\mathrm{WU}, 2005$; Wu et al., 2007). Heritability was the highest for height, intermediate for RYC, and lowest for volume and DBH. Similarly, the highest heritability for height was also observed in other studies on masson pine (HoNG et al., 2010; Du et al., 2010; Ji et al., 2005) and ash (MwASE et al., 2008). In this study, family heritability for height was greater than 0.30 . This was very close to the value from a previous study (0.32; HoNG et al., 2010), but much lower than other published results in which the heritability was 0.69 (Du et al., 2010) and 0.83 (JI et al., 2005), respectively.

The main interests of this study were to examine efficiency of early selection that was based on one-to-one correspondence between early and later heritability. Since the mature ages were different at three sites, only heritabilities from single-site analyses were used for estimation. To our knowledge, there are few publications reporting age-age genetic correlation and early selection of masson pine for growth characteristics and RYC. For most correlations, there was an increasing trend with age. This increasing trend was also observed in $\mathrm{DBH}$ and wood density of radiata pine (LI and WU, 2005; WU et al., 2007; KuMAR and LEE, 2002), scots pine (HAAPANEN, 2001), loblolly pine (BALOCCHI et al., 1994) and Norway spruce (HYLEN, 1999). A correlation drop was observed for $\mathrm{DBH}$ and volume at Yunan. One possible explanation for this drop could be large errors and decreasing population size (GWAZE, 2009).

There was an increasing trend of early selection efficiency with age for most analyses, which was also similar with efficiency for diameter or wood density in radiata pine (LI and WU, 2005; KUMAR and LEE, 2002; Wu et al., 2007), loblolly pine (BALOCCHI et al., 1994) and scots pine (HAAPANEN, 2001). Selection for height at Heyuan was very efficient $(>80 \%)$ and stable at all ages as a result of high genetic correlations and heritabilities. Selection efficiency for height at Xinyi and Yunan improved gradually with the increase in age-age genetic correlations. Selection efficiency for $\mathrm{DBH}$ and volume displayed a similar age trend. Literature suggests that correlations as low as 0.6 could be useful in early selection (NAMKOONG et al., 1980; MwASE, 2008). In this paper, correlation $>0.8$ is considered efficient for early selection, thus selection of masson pine would be effective at age 13 for height, and age 15 for $\mathrm{DBH}$ and volume.

For RYC, selection at age 11 would be highly effective $(>80 \%)$ at three testing sites. Obviously, the high efficiency was closely associated with the high genetic correlations and moderate heritabilities. Moreover, an interesting result was observed that efficiency at three testing sites increased in a similar way at most ages. Selection efficiency was close at age 7 for Xinyi and Heyuan $(0.55 \sim 0.70)$, age 9 for Yunan and Heyuan $(0.80 \sim 0.90)$, and later ages for three test sites $(0.80 \sim 0.90)$. The high and close efficiency at three testing sites strongly suggested that early selection at age 11 for RYC was effective and reliable.

Since the resin tapping is a laborious, time-consuming and destructive procedure, more convenient methodology is needed for RYC measurement. The level of genetic control of a trait and its interrelationships with other traits determine the feasibility of indirect selection in breeding program (ZENG and YUE, 1984). In this study, highly positive genetic correlations were identified between RYC and three growth traits (Table 9), which made the RYC could be easily predicted from three growth traits. $\mathrm{DBH}$ is considered cheaper and easier to measure than height. Both RYC and $\mathrm{DBH}$ are under moderate genetic control (Table 5) and had high age-age genetic correlations and high early selection efficiency (Figures 4 and 5). This suggests that $\mathrm{DBH}$ is a strong candidate trait for indirect genetic improvement of RYC. Additionally, as selection at age 11 would be effective (>80\%) for both RYC and DBH, it is suitable to conduct prediction of RYC at age 11 by using $\mathrm{DBH}$ indirectly. 


\section{Conclusions}

Family and site had significant effects on three growth characteristics (height, DBH and volume) and resin-yielding capacity of masson pine at three testing sites at all ages. The trees showed gradual increase in RYC with age. Xinyi was considered highly suitable for the growth and resin-yielding of masson pine because of rapid growth and high $\mathrm{RYC}$ at later ages. The additive genetic coefficients of variations (CV) for four traits decreased gradually with age. Individual heritability was the highest for height, intermediate for RYC, and lowest for volume and DBH. RYC had highly positive genetic correlations with growth characteristics. Age-age genetic correlations were very high for four traits, and displayed an increasing trend with age. Such high genetic correlations led to effective early selection for four traits.

\section{Acknowledgements}

This work was jointly supported by the Planning Subject of 'the Twelfth Five-Year-Plan' in National Science and Technology for the Rural Development in China (No. 2012BAD01B0202), Forestry Science and Technology Innovation Program of Guangdong Province (No. 2008KJCX005-01) and Ministry and Provincial Industry-university-Institute Strategic Alliance (No. 2007B090400098). The authors thank Prof. BAILIAN LI from North Carolina State University, Dr. HARRY WU from the Commonwealth Scientific and Industrial Research Organisation (CSIRO), and Dr. HUIQUAN ZHENG for paper revision. We also thank an anonymous reviewer for construction comments and suggestions which greatly improved the manuscript.

\section{References}

Balocchi, C. E., F. E. BRidgwater and R. Bryant (1994): Selection efficiency in a nonselected population of loblolly pine. Forest Science 40: 452-473.

BURDON, R. D. (1977): Genetic correlation as a concept for studying genetype-environment interaction in forest tree breeding. Silvae Genetica 26: 5-6.

Coppen, J. J. W. and G. A. Hone (1995): Gum Naval Stores: Turpentine and Resin from Pine Resin, Natural Resources Institute, FAO.

Du, L. F., Y. Chen, Z. F. Ke, D. X. Gao, Y. F. XIE, J. Zou, H. P. LIU, X. H. JIA and Z. W. ZHANG (2010): Genetic gain of analysis on half-sib progenies family of Pinus massoniana. Journal of Huazhong Agricultural University 29: 772-777.

FrIES, A. and T. ERICSSON (2009): Genetic parameters for earlywood and latewood densities and development with increasing age in Scots pine. Annals of Forest Science 66: 404.

Gwaze, D. (2009): Optimum selection age for height in shortleaf pine. New Forests 37: 9-16.

HAAPANEN, M. (2001): Time trends in genetic parameters estimates and selection efficiency for scots pine in relation to field testing method. Forest Genetics 8: 129-144.

He, B. X., H. M. Lian, L. H. Zeng and Z. C. Ruan (1999): A study on pine resin chemical composition and its geographic variation of high resin masson pine superior tree. Guangdong Forestry Science and Technology 15: 1-7.
Hong, Y. H., W. J. LIN and Y. F. HUANG (2010): Selection of excellent families and analysis on growth variation for the 12-year-old half-sib family of seed orchard of Pinus massoniana. Journal of Nanjing Forestry University 34: 26-31.

Hylen, G. (1999): Age trends in genetic parameters of wood density in young Norway spruce. Canadian Journal Forest Research 29: 135-143.

JI, K. S., M. L. FAN and L. A. XU (2005): Variation analysis and plus family selection on half-sib progenies from clonal seed orchard of Pinus massoniana. Scientia Silvae Sinicae 41: 43-49.

JiN, G. Q., G. F. QIN, W. H. LiU, D. Y. ChU, S. Z. Hong and Z. C. ZHOU (2008a): Effects of mating manners on growth traits of Pinus massoniana and selection of cross combinations. Scientia Silvae Sinicae 44: 29-35.

JIN, G. Q., G. F. QIN, W. H. LiU, D. Y. CHU, S. Z. Hong and Z. C. ZHOU (2008b): Genetic analysis of growth traits on tester strain progeny of Pinus massoniana. Scientia Silvae Sinicae 44: 71-79.

KumAR, S. and J. LeE (2002): Age-age correlations and early selection for end-of-rotation wood dentsity in radiata pine. Forest Genetics 9: 323-330.

LEDIG, F. T. (1974): Analysis of methods for the selection of trees from wild stands. Forest Science 20: 2-16.

LI, L. and H. X. WU (2005): Efficiency of early selection for rotation-aged growth and wood density traits in Pinus radiata. Canadian Journal of Forest Research 35: 2019-2029.

Lian, H. M., L. H. Zeng, B. X. He, M. Luo, J. QIn, Z. Y. QI and R. K. LUO (2006): Study on the genetic variation of wood properties and correlation in wood properties and growth, resin yielding ability. Guangdong Forestry Science and Technology 22: 5-11.

Lin, Q. H., R. Zhang, G. Q. Jin, D. Y. Chu and Z. C. Zhou (2010): Variation of ring width and wood basic density and early selection of Pinus massoniana provenances. Scientia Silvae Sinicae 46: 50-56.

LiU, Q. H., G. Q. Jin, R. Zhang, D. Y. ChU, G. F. QIN and Z. C. ZHOU (2009): Provenance variation in growth, stem-form and wood density of masson pine at 24-yearold and the provenance division. Scientia Silvae Sinicae 45: $55-62$

LIU, Y. (2001): Production, consumption and prediction of China's resin. Journal of Chemical Industry of Forest products 35: 31-33.

Mwase, W. F., P. S. Savill and G. Hemery (2008): Genetic parameter estimates for growth and form traits in common ash (Fraxinus excelsior, L.) in a breeding seedling orchard at Little Wittenham in England. New Forests 36: $225-238$.

Namkoong, G., R. D. Barnes and J. Burley (1980): A philosophy of breeding strategy for tropical forest trees. Tropical Forestry Paper No. 16. Commonwealth Forestry Institute, Oxford, $67 \mathrm{pp}$.

Sebbenn, A. M., F. C. Arantes, O. V. Boas and M. L. M. FREITAS (2008): Genetic variation in an international provenance-progeny test of Pinus caribaea Mor. var. bahamensis Bar. et Gol., in Sao Paulo, Brizil. Silvae Genetica 57: 181-187.

SHI, K. and Z. Li (1998): The Development of China's Forestry: Review and Prospects. The Environmental Science Press of China, Beijing. pp 110.

Tomusiak, R. and M. MAGNUSZEWSKI (2009): Effect of resin tapping on radial increments of Scots pine (Pinus sylvestris L.). In: KACZKA, R., I. MALIK, P. OWCZARE, H. Gärtner, G. Helle and I. Heinrich (eds.) (2009): 